\title{
Fingerprint Enhancement using Improved Orientation and Circular Gabor Filter
}

\author{
Avinash Pokhriyal \\ FMCA, R.B.S. College, Agra- \\ 282002, India
}

\author{
Praveen Sengar \\ FMCA, R.B.S. College, Agra- \\ 282002, India
}

\author{
Sushma Lehri \\ IET, Dr. B. R. Ambedkar \\ University, Agra-282002, India
}

\begin{abstract}
In an automatic fingerprint identification system (AFIS), the fingerprint enhancement algorithm is mainly used to improve the visual quality of the input fingerprint image. The factors affecting the quality of an input fingerprint image may be the presence of scars, cuts, pressure variation between the finger and sensor, worn artifacts, and humidity during acquisition process. An enhancement algorithm is applied on the input fingerprint image to improve image quality and to repair broken ridges.

This paper proposes a new method of enhancing fingerprint image. The method uses a hybrid approach of a new orientation scheme and a Circular Gabor filter to improve the visual quality of the fingerprint image. The proposed approach is compared to other enhancement algorithms using structural similarity index (SSIM). Experimental results and comparative analysis show that the new method performs better than the previous methods .
\end{abstract}

\section{Keywords}

Fingerprint image, Histogram Equalization, Fast Fourier Transform, Orientation, Circular Gabor filter, SSIM.

\section{INTRODUCTION}

Fingerprint is a pattern of ridges and valleys in a finger, which is unique and permanent in every human being. Fingerprints have some unique features by which they are distinguished from each other. There are three types of features found in a fingerprint: level-1 features (core and delta), level 2 features (minutiae), and level 3 features (sweat pores). Out of these features, minutiae are the mostly used features for recognizing fingerprints because they mimic the way human experts recognize fingerprints. Minutiae are minute details present in the fingerprint in the form of points that either abruptly end (terminations) or fork into two branches deriving from a point (bifurcations).

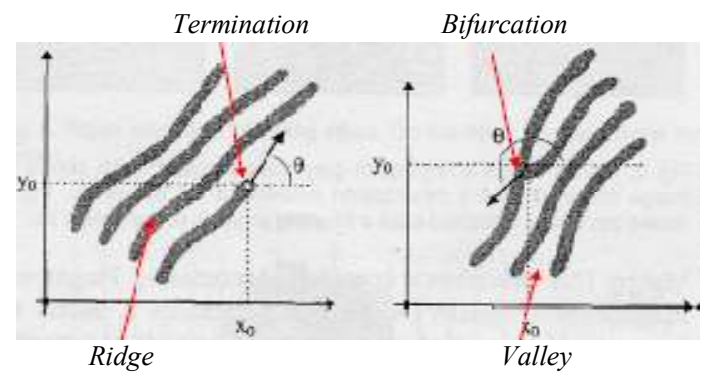

Figure 1. Minutiae: Termination \& Bifurcation

\section{FINGERPRINT ENHANCEMENT}

Fingerprint enhancement is a process to improve the clarity of ridge structures in a fingerprint image and filling broken ridges so that genuine minutiae could be extracted at the later stage. The enhancement process should not produce any false ridges as they may change the individuality of input fingerprints. Since the fingerprint images acquired from sensors or other media are not of perfect quality, some enhancement process is needed to increase the contrast between ridges and valleys and to connect the ridges broken due to insufficient amount of ink. Enhance-ment is useful for higher accuracy of the fingerprint recognition. There have been several enhancement algorithms devised so far [1-8], but still there is enough space for improvement in finger-print image quality.

\subsection{Histogram Equalization}

Histogram equalization [1] is to expand the pixel value distribution of an image so as to increase the perceptional information. The original histogram of a fingerprint image has the bimodal type, the histogram after the histogram equalization occupies all the range from 0 to 255 and the visualization effect is enhanced. Figure 2 depicts the original image and the enhanced image after histogram equalization.

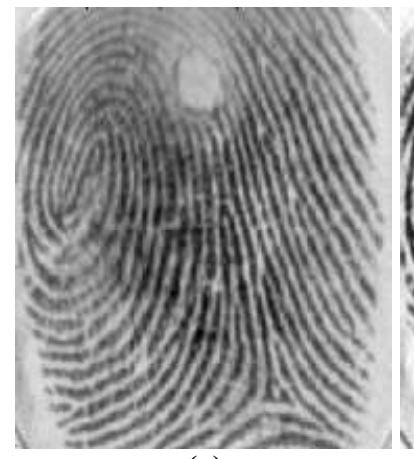

(a)

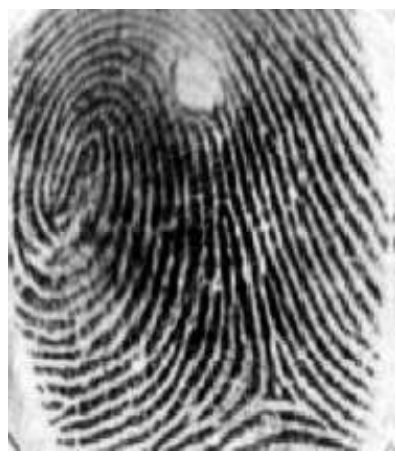

(b)
Figure 2. (a) Original image (b) histogram-equalized image

\subsection{FFT-Based Enhancement}

Cikkerur et al. [5] proposed Fast Fourier Transform (FFT) based enhancement of the fingerprint image. The algorithm is given as follows:

Divide the image into small processing blocks ( 32 x 32 pixels) and perform the Fourier transform according to: 


$$
\begin{gathered}
F(u, v)=\sum_{x=0}^{M-1} \sum_{y=0}^{N-1} f(x, y) e^{-j .2 \pi\left(\frac{u x}{M}+\frac{v y}{N}\right)} \\
u, v=0,1,2, \ldots, 31
\end{gathered}
$$

To enhance a specific block by its dominant frequencies, multiply FFT of the block by its magnitude a set of times as:

$$
g(x, y)=F^{-1}\left\{F(u, v) .|F(u, v)|^{k}\right\}
$$

where $F^{-1}(F(u, v))$ is given as:

$$
f(x, y)=\frac{1}{M N} \sum_{x=0}^{M-1} \sum_{y=0}^{N-1} F(u, v) e^{-j .2 \pi\left(\frac{u x}{M}+\frac{v y}{N}\right)}
$$

$$
\text { for } x, y=0,1,2, \ldots, 31
$$

We take $k=0.43$ in the formula (2). A higher value of " $k$ " may improve the appearance of the ridges by filling up small holes in ridges, but too high a " $k$ " may result in false joining of ridges causing a termination to become a bifurcation or vice-versa. An FFT-based fingerprint enhancement is depicted in figure 3.

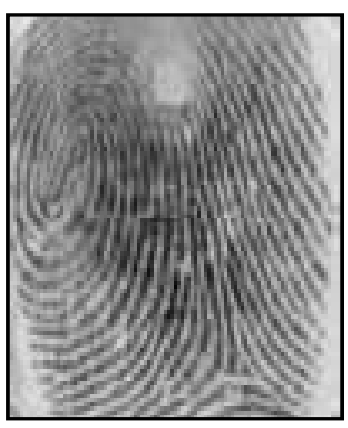

(a)

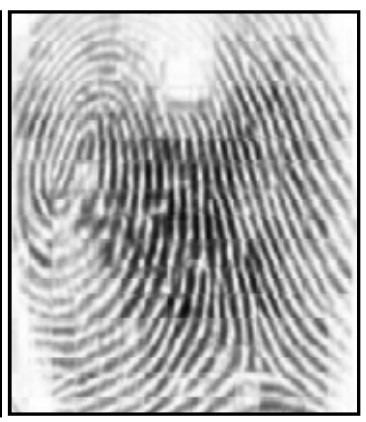

(b)
Figure 3. (a) Original image (b) FFT-enhanced image

\subsection{Gabor-filter Based Enhancement}

Hong et al. [3, 4] used Gabor filter for fingerprint enhancement, which has both frequency-selective and orientation-selective properties. Gabor-filtering an image demands 3 parameters: (a) frequency $f$ of the sinusoidal wave, determined by the local ridge frequency (b) the filter orientation $O$, determined by the local ridge orientation, and (c) the standard deviations of the Gaussian envelope $-\sigma_{x}, \sigma_{y}$. Hong et al.'s algorithm uses even-symmetric square Gabor filter defined as the real part of the Gabor function given by a cosine wave modulated by a Gaussian

$$
\begin{aligned}
& h(x, y: \emptyset, f)=e^{-\frac{1}{2}\left[\frac{x_{\emptyset}^{2}}{\sigma_{x}^{2}}+\frac{y_{\phi}^{2}}{\sigma_{y}^{2}}\right]} \cos \left(2 \pi f x_{\emptyset}\right) \\
& x_{\varnothing}=x \cos \emptyset+y \sin \emptyset \\
& y_{\emptyset}=-x \sin \emptyset+y \cos \emptyset
\end{aligned}
$$

Here, $\varnothing$ is the orientation of the Gabor filter, $f$ is the frequency of a sinusoidal plane wave, $\sigma_{x}$ and $\sigma_{y}$ the standard deviations of the Gaussian envelope along $\mathrm{x}$ and $\mathrm{y}$ axes, respectively, and $x_{\varnothing}$ and $y_{\varnothing}$ the $\mathrm{x}$ and $\mathrm{y}$ axes of the filter coordinate frame. This square Gabor filter is applied to the fingerprint image by spatially convolving the image with the filter. The convolution of a pixel $(i, j)$ in the image requires the corresponding orientation value $O(i, j)$ and ridge frequency value $F(i, j)$ of that pixel. Let $\mathrm{G}$ be the normalized fingerprint image, and $R$ be the recoverable mask, then enhanced image $E$ is obtained as:

$E(i, j)=$

$255 \quad$,if $R(i, j)=0$

$\left\{\sum_{u} \sum_{v} h(u, v: O(i, j), G(i-u, j-v), \quad\right.$ else

where, $-w_{g} / 2 \leq u, v \leq w_{g} / 2$, and $w_{g}$ specifies the size of the Gabor filters. The problem with HWJ approach is that the standard deviation of the Gaussian envelope $\left(\sigma_{x}, \sigma_{y}\right)$ are empirically selected, computational complexity, filter size selection depends on the frequency feature of the image, and it doesn't work well for noisy images.

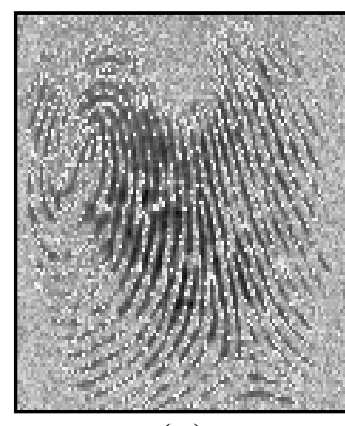

(a)

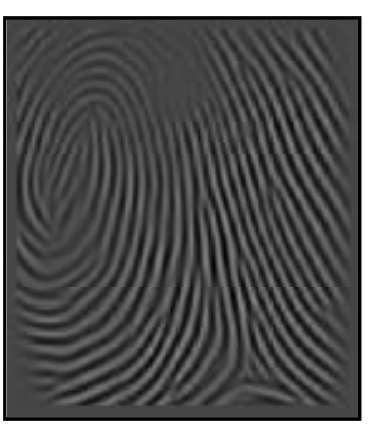

(b)
Figure 4. (a) Original image (b) Gabor-filter enhanced image

The Hong et al. [6] orientation estimation algorithm is, 1. Normalize the input image I to get normalized image N. 2. Divide N into wxw blocks; centre each block B at N(i,j) 3. At each pixel $N(i, j)$, compute gradients $\partial_{x}(i, j) \& \partial_{y}(i, j)$ 4. Compute $V_{\mathrm{x}}$ and $\mathrm{V}_{\mathrm{y}}$ as,

$$
\begin{aligned}
& V_{x}(i, j)=\sum_{u=i-\frac{w}{2}}^{i+\frac{w}{2}} \sum_{v=j-\frac{w}{2}}^{j+\frac{w}{2}} 2 \partial_{x}(u, v) \partial_{y}(u, v) \\
& V_{y}(i, j)=\sum_{u=i-\frac{w}{2}}^{i+\frac{w}{2}} \sum_{v=j-\frac{w}{2}}^{j+\frac{w}{2}} \partial_{x}{ }^{2}(u, v) \partial_{y}{ }^{2}(u, v)
\end{aligned}
$$

5. Find local orientation $\theta$ of $B$ as:

$$
\theta(\mathrm{i}, \mathrm{j})=\frac{1}{2} \tan ^{-1} \frac{\mathrm{V}_{\mathrm{y}}(\mathrm{i}, \mathrm{j})}{\mathrm{V}_{\mathrm{x}}(\mathrm{i}, \mathrm{j})}
$$

6. $\theta(\mathrm{i}, \mathrm{j})$ is smoothened (as it may be noisy) by

(i) first converting it into a continuous vector field.

$$
\begin{aligned}
& \Phi_{x}(\mathrm{i}, \mathrm{j})=\cos (2 \theta(\mathrm{i}, \mathrm{j})) \\
& \Phi_{\mathrm{y}}(\mathrm{i}, \mathrm{j})=\sin (2 \theta(\mathrm{i}, \mathrm{j})) \\
& \Phi_{\mathrm{x}}, \Phi_{\mathrm{y}} \text { are } \mathrm{x} \text { and } \mathrm{y} \text { components of the vector field. }
\end{aligned}
$$

(ii) performing Gaussian smoothening at the block level.

$$
\begin{aligned}
& \Phi^{\prime}{ }_{X}(\mathrm{i}, \mathrm{j})=\sum_{\mathrm{u}} \sum_{\mathrm{v}} \mathrm{G}(\mathrm{u}, \mathrm{v}) \emptyset_{\mathrm{x}}(\mathrm{i}-\mathrm{uw}, \mathrm{j}-\mathrm{vw}) \\
& \Phi^{\prime}{ }_{\mathrm{Y}}(\mathrm{i}, \mathrm{j})=\sum_{\mathrm{u}} \sum_{\mathrm{v}} \mathrm{G}(\mathrm{u}, \mathrm{v}) \emptyset_{\mathrm{y}}(\mathrm{i}-\mathrm{uw}, \mathrm{j}-\mathrm{vw})
\end{aligned}
$$


where, $\frac{\mathrm{w}_{\emptyset}}{2} \leq \mathrm{u}, \mathrm{v} \leq \frac{\mathrm{w}_{\emptyset}}{2}$, and $\mathrm{G}$ is a Gaussian low-pass filter of size $\mathrm{w}_{\boldsymbol{\Phi}} \mathrm{X} \mathrm{w}_{\boldsymbol{\Phi}} .\left(\mathrm{w}_{\boldsymbol{\Phi}}=5\right)$.

7. finally, the smoothed orientation $\mathrm{O}$ at pixel $(i, j)$ is computed:

$\mathrm{O}(\mathrm{i}, \mathrm{j})=\frac{1}{2} \tan ^{-1} \frac{\phi_{\mathrm{y}}(\mathrm{i}, \mathrm{j})}{\hat{\emptyset}_{\mathrm{x}}(\mathrm{i}, \mathrm{j})}$

Hong et al.'s method of estimating orientation map is simple to implement and has a high resolution, but the averaging of the doubled trigonometrically calculated values has high computa-tional cost and the large areas of heavy corrupted data still affect final orientation values. The orientation map is rough in case of noisy images or images with ridge breaks. An orientation map obtained using hong et al.'s approach [6] is depicted in Figure 5.

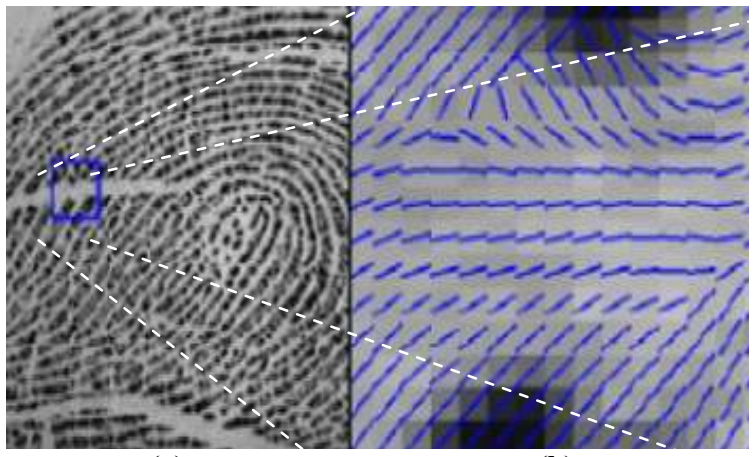

(a)

(b)

Figure 5: a) Original low quality fingerprint image; b) magnified area with corrupted orientation values marked

Another problem area of Hong et al.'s approach is square Gabor filter that enhances different blocks with different frequencies and directions, to different clarities. This creates a blocky effect due to the filter's square support (see Figure 6).

\section{FINGERPRINT ENHANCEMENT USING IMPROVED ORIENTATION AND CIRCULAR GABOR FILTER}

We introduce a new fingerprint enhancement technique that takes on a hybrid approach of improving orientation map as well as filtering the image using circular Gabor filter.

First, we improve the orientation map obtained by Hong et al.'s algorithm[6]. The method used here tries to improve orientation

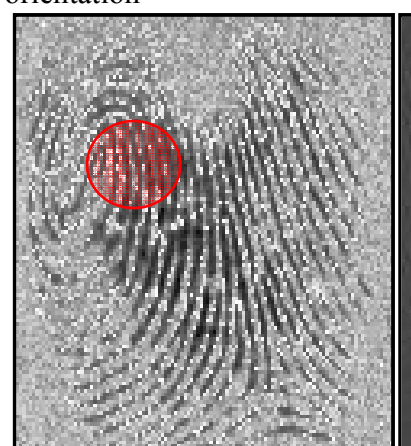

(a)

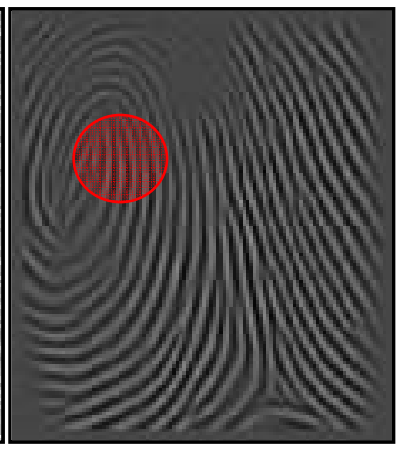

(b)
Figure 6: a) Original image ; b) Gabor-filtered image. The blocky effect depicted by red circle in both the images. estimation in heavy noise and broken-ridge areas. It also tries to preserve the orientation values of singular points area. This is a two-step process:

Step-I: a 2-D low-pass Gaussian is used to modify the rough local ridge orientation. The weighted averaging is computed for two ranges of orientation values separately. For $45^{\circ}>\theta(u$, $v)>135^{\circ}$, the vertical weight $W_{v}$, and the vertical weighted mean factors $\mathrm{M}_{v}(i, j)$ in $\mathrm{w} \times \mathrm{w}$ blocks is computed as,

$$
\begin{gathered}
\mathrm{W}_{v}(i, j)=\sum_{\mathrm{u}} \sum_{\mathrm{v}} \partial(u, v) G(u-i, v) \\
\mathrm{M}_{v}(i, j)=\sum_{\mathrm{u}} \sum_{\mathrm{v}}(\theta(u, v)-z) \partial(u, v) G(u-i, v \\
-j)
\end{gathered}
$$

$z$

$$
=\left\{\begin{array}{rr}
0, & \text { if } \theta(i, j)<45^{\circ} \\
\pi, & \text { otherwise }
\end{array}\right.
$$

and, for $45^{\circ} \leq \theta(u, v) \leq 135^{\circ}$, horizontal weight $W_{h}$, and horizontal weighted mean factors $\mathrm{M}_{h}(i, j)$ in wxw blocks is computed as,

$$
\begin{aligned}
& \mathrm{W}_{h}(i, j)=\sum_{\mathrm{u}} \sum_{\mathrm{v}} \partial(u, v) G(u-i, v-j) \\
& \mathrm{M}_{h}(i, j)=\sum_{\mathrm{u}} \sum_{\mathrm{v}} \theta(u, v) \partial(u, v) G(u-i, v-j)
\end{aligned}
$$

Finally filtered orientation is calculated using weighted mean:

$$
\begin{aligned}
& \quad= \begin{cases}\frac{\mathrm{M}_{v}(i, j) \mathrm{W}_{v}(i, j)+\left(\mathrm{M}_{h}(i, j)+\Pi\right) \mathrm{W}_{h}(i, j)}{\mathrm{W}_{v}(i, j)+\mathrm{W}_{h}(i, j)}, & \text { if } M_{d}(i, j)>\frac{\pi}{2} \\
\frac{\mathrm{M}_{v}(i, j) \mathrm{W}_{v}(i, j)+\mathrm{M}_{h}(i, j) \mathrm{W}_{h}(i, j)}{\mathrm{W}_{v}(i, j)+\mathrm{W}_{h}(i, j)}, & \text { if } M_{d}(i, j) \leq \frac{\pi}{2}\end{cases} \\
& M_{d}(i, j)=M_{v}(i, j)-M_{h}(i, j) \\
& \text { where, } \quad i-\frac{w}{2} \leq u, v \leq i+\frac{w}{2}
\end{aligned}
$$

Step-II: The orientation smoothing is based on the reliability of estimated orientation that corresponds to differentiation values of neighboring pixels. A larger neighborhood $(16 \times 16)$ is used to compute the differentiation values:

$$
\begin{aligned}
& D(i, j)=\sum_{u} \sum_{v}|O(i, j)-o(u, v)|-z \\
& \text { where, } \quad i-\frac{w}{2} \leq u, v \leq i+\frac{w}{2} \\
& z=\left\{\begin{array}{lr}
\pi, & \text { if }|O(i, j)-O(u, v)|>90^{\circ} \\
0, & \text { otherwise }
\end{array}\right.
\end{aligned}
$$

Finally, the entire first stage is performed once again, but now the Gaussian filter $G(x, y)$ is replaced by multiplicative inverse of differentiation, i.e. 


$$
G(x, y)=\frac{1}{D(x, y)}
$$

The zoomed portions of the fingerprints with cuts and noise in figure 3 show that this new approach to smooth the oriented fingerprint image has very pleasing results.

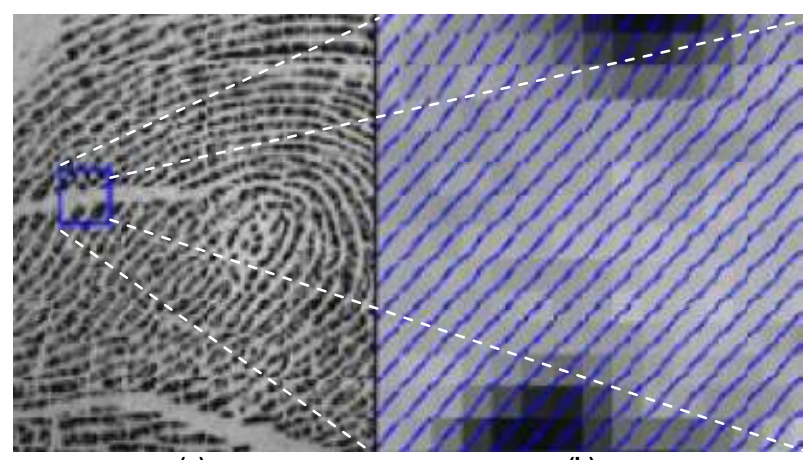

(a)

(b)

Figure 7: a) low quality fingerprint image; b) magnified area with improved orientation values marked

In order to remove the blocky effect, we use a circular Gabor filter (CGF) with the diameter adjusted to trice the ridge width just crossing one ridge and two valleys (Figure 2) defined as:

$$
\begin{gathered}
h^{\prime}(x, y: \emptyset, f)=\left\{\begin{array}{cl}
h(x, y: \emptyset, f) & \text { if } x^{2}+y^{2} \leq \tau^{2} \\
0 & \text { otherwise }
\end{array}\right. \\
\quad \text { Where, } \\
\tau=\frac{3}{4} T
\end{gathered}
$$

$\mathrm{T}$ being the average distance between two peaks.

The enhanced image $E$ ' using the CGF is obtained as:

if $R\left(\left\lfloor\frac{i}{w}\right\rfloor,\left\lfloor\frac{j}{w}\right\rfloor\right)=0$

then $\left.E^{\prime}(i, j)=\sum_{u} \sum_{v} h^{\prime}\left(u, v: O\left(\left\lfloor\frac{i}{w}\right\rfloor, \mid \frac{j}{w}\right\rfloor\right), f\right) G\left(i^{\prime}, j^{\prime}\right)$

else $E^{\prime}(i, j)=255$

where $i^{\prime}=i-u, j^{\prime}=j-v$, and $-\tau \leq u, v \leq \tau$

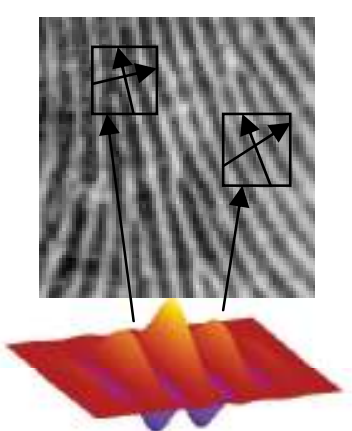

(a)

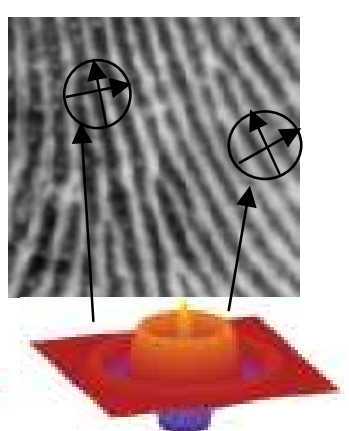

(b)
Figure 8. (a) square Gabor filter (b) Circular Gabor filter The effect of improving the orientation and filtering by CGF enhances blocks with different orientations to the same clarity.

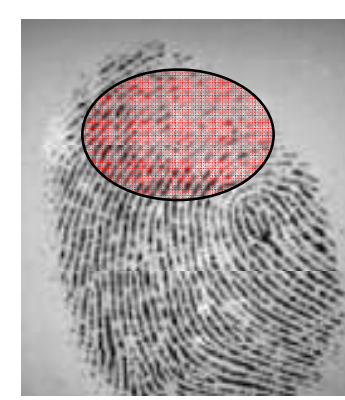

(a)

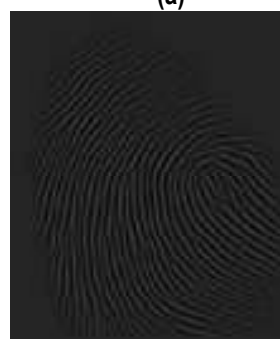

(c)

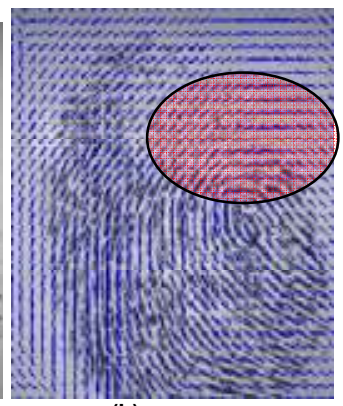

(b)

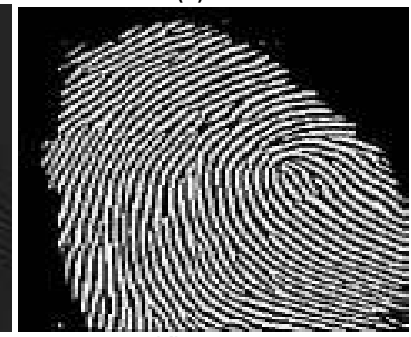

(d)
Figure 9 a) Noisy fingerprint image with hedgy ridges marked; b) Improved Orientation; c) Enhanced image; d) binary image by proposed method. The highlighted portion has hedgy ridges that are repaired by improved orientation in (b) and CGF filtering in (c). the affect of this approach is clearly seen in the binary image (d) that shows the hedgy ridges have been repaired.

Figure shows the effect of the proposed method on a noisy fingerprint image taken from FVC2000 DB1 database.

\section{EXPERIMENTAL RESULTS AND COMPARISONS}

Though there are many methods to assess fingerprint image quality (like SNR, PSNR, MSE, etc.), but structural similarity (SSIM) index proposed by Wang et al. $[8,9,10]$ proves to be the best among them. The structural similarity between the input fingerprint image $\mathrm{x}$ and enhanced image $\mathrm{y}$ is given as,

$$
\operatorname{SSIM}(x, y)=\frac{\left(2 \mu_{x} \mu_{y}+c_{1} L\right)\left(2 \sigma_{x y}+c_{2} L\right)}{\left(\mu_{x}^{2}+\mu_{y}^{2}+c_{1} L\right)\left(\sigma_{x}^{2}+\sigma_{y}^{2}+c_{2} L\right)}
$$

$\mu_{x}, \mu_{y}$ are means and $\sigma_{x}^{2}, \sigma_{y}^{2}$ are variances of $x$ and $y$ respective-ly, $\sigma_{x} \sigma_{y}$ is covariance of $x$ and $y, c_{1}, c_{2}$ are adjustable constants, and $L$ is the maximal possible value of $x$. Different fingerprint images from FVC2002 DB1 database were taken for assessing the SSIM index between original and enhanced images was observed as shown in the table 1 .

The comparison chart based on SSIM indices given in table is shown in figure 5 . The chart shows the proposed enhancement scheme in sky blue color, which clearly shows that the proposed scheme's similarity indices attain the maximum possible values against other schemes. This clearly indicates that the proposed scheme enhances given input fingerprint images in a better way as compared to other schemes. 
Table 1. SSIM index of fingerprint images (FVC2002) for different enhancement schemes. Proposed scheme clearly shows the best indices (for all the fingerprint images) among all the four schemes.

\begin{tabular}{|l|l|l|l|l|}
\hline $\begin{array}{l}\text { Fingerprint } \\
\text { image }\end{array}$ & $\begin{array}{l}\text { Histogram } \\
\text { based }\end{array}$ & $\begin{array}{l}\text { FFT- } \\
\text { based }\end{array}$ & $\begin{array}{l}\text { Gabor } \\
\text { based }\end{array}$ & Proposed \\
\hline 101_1.tif & 0.98455 & 0.99033 & 0.99189 & 0.99684 \\
\hline 101_2.tif & 0.98546 & 0.99381 & 0.99269 & 0.99753 \\
\hline 101_3.tif & 0.98151 & 0.99397 & 0.99045 & 0.99742 \\
\hline 101_4.tif & 0.98991 & 0.99117 & 0.99356 & 0.99611 \\
\hline 101_5.tif & 0.99188 & 0.99240 & 0.99414 & 0.99591 \\
\hline 101_6.tif & 0.98346 & 0.99574 & 0.99065 & 0.99847 \\
\hline 101_7.tif & 0.98783 & 0.99029 & 0.99559 & 0.99500 \\
\hline 101_8.tif & 0.98195 & 0.99254 & 0.99358 & 0.99510 \\
\hline 102_1.tif & 0.99370 & 0.99181 & 0.99540 & 0.99507 \\
\hline 102_2.tif & 0.99044 & 0.99261 & 0.99436 & 0.99590 \\
\hline 102_3.tif & 0.99110 & 0.99319 & 0.99469 & 0.99566 \\
\hline 102_4.tif & 0.98861 & 0.99451 & 0.99275 & 0.99791 \\
\hline 102_5.tif & 0.99243 & 0.99228 & 0.99183 & 0.99496 \\
\hline 102_6.tif & 0.99081 & 0.99159 & 0.99404 & 0.99701 \\
\hline 102_7.tif & 0.99060 & 0.99449 & 0.99541 & 0.99688 \\
\hline 102_8.tif & 0.99114 & 0.99090 & 0.99240 & 0.99422 \\
\hline 103_1.tif & 0.98895 & 0.99139 & 0.99331 & 0.99622 \\
\hline 103_2.tif & 0.99065 & 0.98984 & 0.99261 & 0.99522 \\
\hline 103_3.tif & 0.98946 & 0.99054 & 0.99253 & 0.99685 \\
\hline 103_4.tif & 0.98926 & 0.99129 & 0.99234 & 0.99665 \\
\hline 103_5.tif & 0.98902 & 0.99403 & 0.98972 & 0.99824 \\
\hline 103_6.tif & 0.98709 & 0.99427 & 0.99082 & 0.99844 \\
\hline 103_7.tif & 0.98866 & 0.99372 & 0.99080 & 0.99826 \\
\hline 103_8.tif & 0.98696 & 0.99449 & 0.98879 & 0.99836 \\
\hline & & & & \\
\hline
\end{tabular}

\section{CONCLUSIONS}

The paper proposes a new way of enhancing the input fingerprint images, and compares the proposed scheme with previous enhancing schemes such as histogram equalization, FFT-based enhancement, and Gabor-filter based enhancement. The comparative study shows that the proposed scheme produces the maximum structural similarity indices in most of the cases, which means that it enhances the fingerprint in such a way that preserves the image's pattern and also enhances it in a nice manner. So the proposed method of enhancement is better than its previous counterparts.

\section{REFERENCES}

[1] Ahmed Wathik Naji, Abd Rahman Ramli, Rosaliza Ali, Syed Abdul Rahman, Mohd. Liakot Ali, A Segmentation Algorithm based-on Histogram Equalizer For Fingerprint Classification System, ICECE 2002, 26-28 December 2002

[2] R. Thai. Fingerprint Image Enhancement and Minutiae Extraction. PhD thesis, School of Computer Science and Software Engineering, 2003.

[3] Shlomo Greenberg, Mayer Aladjem, Daniel Kogan, and Itshak Dimitrov. Fingerprint image enhancement using Filtering techniques. In International Conference on Pattern Recognition,pages $322\{325$. IEEE, 2000.

[4] Yuliang He, Jie Tian, Xiping Luo, and Tanghui Zhang. Image enhancement and minutiae matching in fingerprint verification. Pattern Recognition Letters, 24:1349\{1360, 2002
[5] Sharat S. Chikkerur, Alexander N. Cartwright and Venu Govindaraju, Fingerprint Image Enhancement Using Stft Analysis, Pattern Recogn. 40, 1 (Jan. 2007), 198-211.

[6] Lin Hong, Yifei Wan, and Anil Jain, Fingerprint Image Enhance-ment: Algorithm and Performance Evaluation, IEEE Transactions On Pattern Analysis And Machine Intelligence, Vol. 20, No. 8

[7] Chaohong Wu, Zhixin Shi, and Venu Govindaraju. Fingerprint image enhancement method using directional median filter, in biometric technology for human identification. Proceedings of the SPIE, pages $250\{256$, 2004.

[8] D. Maltoni, D. Maio, A.K. Jain and S. Prabhakar, Handbook of Fingerprint Recognition, Springer, New York, 2009

[9] Z. Wang and A. C. Bovik, "A universal image quality index," IEEE Signal Process. Lett., vol. 9, no. 3, pp. 8184, Mar. 2002.

[10] Z. Wang and A. C. Bovik, Modern Image Quality Assessment. San Rafael, CA: Morgan and Claypool, 2006.

[11] Z. Wang, A. C. Bovik, H. R. Sheikh, and E. P. Simoncelli, "Image quality assessment: From error visibility to structural similarity," IEEE Trans. Image Process., vol. 13, no. 4, pp. 600-612, Apr. 2004.

\section{AUTHORS PROFILE}

Avinash Pokhriyal received the degree in Master of Computer Applications from Dr. B.R. Ambedkar University, Agra,UP, India in 1997. He is doing his Ph.D. on the topic Minutiae based Automatic Fingerprint Recognition. Currently, he is Reader in MCA department at Faculty of Management \& Computer Application, RBS college, Agra, UP, India. He has published and presented several papers in national and international journals and conferences. He has reviewed many papers in several reputed journals such as IJCSNS, JISE, JITE, WASET, IJCSES, etc. He is a member of Editorial board of reputed international journals like IJoFCS, IJCA, IJBB, IACSIT, IJPS, etc. His research interests include biometric based recognition, image processing, pattern recognition, soft computing, etc.

Prvaeen Sengar received the degree in Master of Computer Applications from Dr. B.R. Ambedkar University, Agra,UP, India in 1997. Currently, he is Assistant Professor in MCA department at Faculty of Management \& Computer Application, R.B.S. College, Agra, UP, India. He has published many papers in several reputed journals like Journal of IAPS, IJBB, etc. His research interests include image processing, pattern recognition, multimedia applications, etc.

Sushma Lehri received the M.Tech. degree in Computer Science from the DOEACC, India in 2000. She received the Ph.D. degree in Solid State Physics from the Dr. B.R. Ambedkar University, Agra, UP, India in 1980. Currently, she is Head \& Associate professor at Institute of Computer \& Information Sciences of Dr. B. R. Ambedkar University. She has many publications in national \& international journals. She has many awards such as research fellowship and postdoctoral fellowship by university grant commission (UGC), New Delhi to her name. Her research interests include image processing, network security, and solid state physics. 


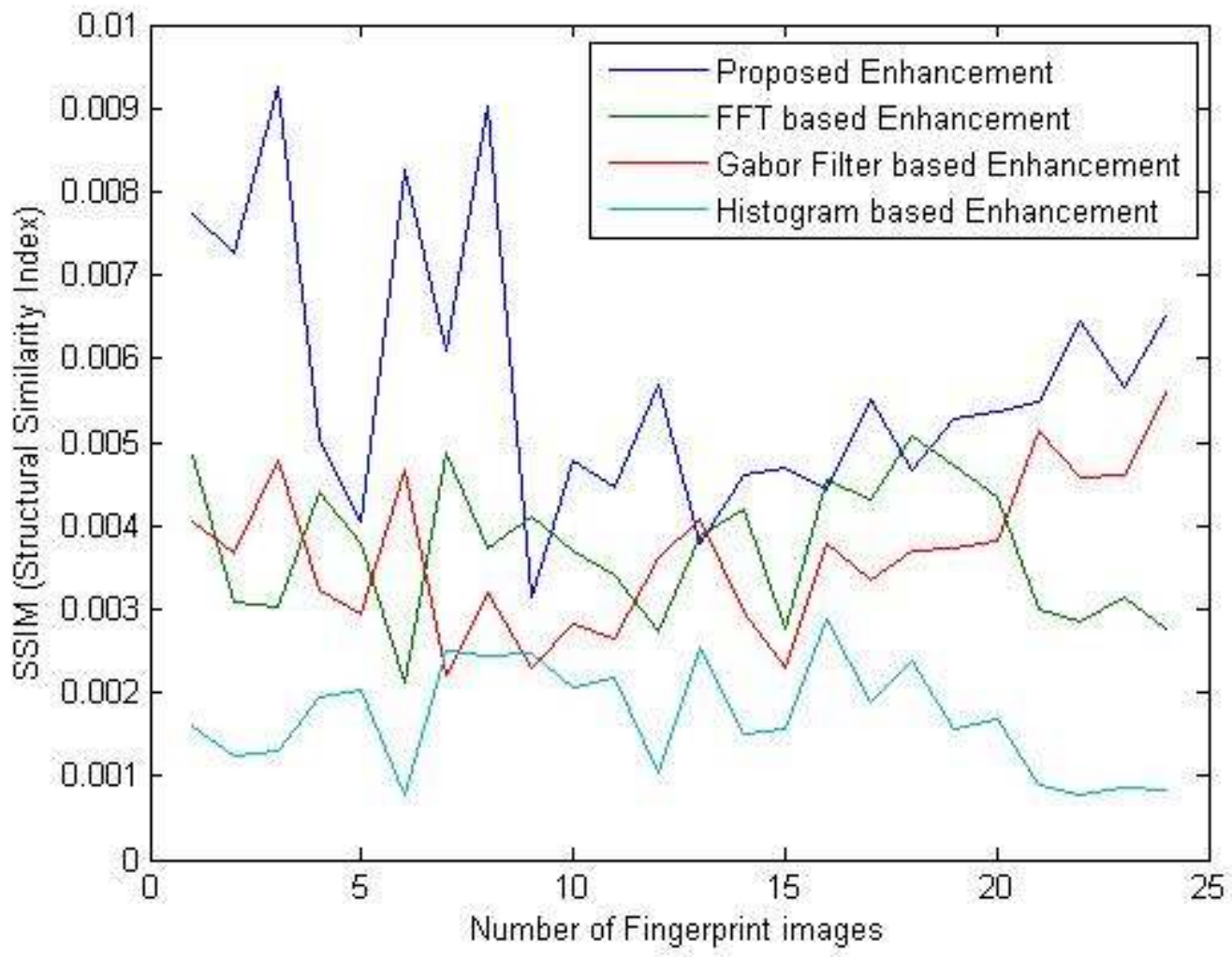

Figure 10. Comparison chart of histogram-based, FFT-based, Gabor filter based, and proposed enhancement schemes 